\title{
Lipopolysaccharide exposure induces oxidative damage in Caenorhabditis elegans: protective effects of carnosine
}

Jing $\mathrm{Ma}^{1+}$, Xiaoyuan $\mathrm{Xu}^{2+}$, Ranran Wang ${ }^{1}$, Haijing Yan ${ }^{3}$, Huijuan Yao ${ }^{1}$, Hongmei Zhang ${ }^{2}$, Shaowei Jiang ${ }^{4^{*}}$ and Ajing $X u^{1 *}$ (iD

\begin{abstract}
Background: The present study was designed to investigate the protective effects and mechanisms of carnosine on lipopolysaccharide (LPS)-induced injury in Caenorhabditis elegans.

Methods: C. elegans individuals were stimulated for $24 \mathrm{~h}$ with LPS $(100 \mu \mathrm{g} / \mathrm{mL})$, with or without carnosine $(0.1,1$, $10 \mathrm{mM}$ ). The survival rates and behaviors were determined. The activities of superoxide dismutase (SOD), glutathione reductase (GR), and catalase (CAT) and levels of malondialdehyde (MDA) and glutathione (GSH) were determined using the respective kits. Reverse transcription polymerase chain reaction (RT-PCR) was performed to validate the differential expression of sod-1, sod-2, sod-3, daf-16, ced-3, ced-9, sek-1, and pmk-1. Western blotting was used to determine the levels of SEK1, p38 mitogen-activated protein kinase (MAPK), cleaved caspase3, and Bcl-2. C. elegans sek-1 (km2) mutants and pmk-1 (km25) mutants were used to elucidate the role of the p38 MAPK signaling pathway.

Results: Carnosine improved the survival of LPS-treated C. elegans and rescued behavioral phenotypes. It also restrained oxidative stress by decreasing MDA levels and increasing SOD, GR, CAT, and GSH levels. RT-PCR results showed that carnosine treatment of wild-type C. elegans up-regulated the mRNA expression of the antioxidantrelated genes sod-1, sod-2, sod-3, and daf-16. The expression of the anti-apoptosis-related gene ced-9 and apoptosisrelated gene ced-3 was reversed by carnosine. In addition, carnosine treatment significantly decreased cleaved caspase3 levels and increased BCl-2 levels in LPS-treated C. elegans. Apoptosis in the loss-of-function strains of the p38 MAPK signaling pathway was suppressed under LPS stress; however, the apoptotic effects of LPS were blocked in the sek-1 and pmk-1 mutants. The expression levels of sek-1 and pmk-1 mRNAs were up-regulated by LPS and reversed by carnosine. Finally, the expression of p-p38MAPK and SEK1 was significantly increased by LPS, which was reversed by carnosine.

(Continued on next page)
\end{abstract}

\footnotetext{
* Correspondence: jiangshaowei@xinhuamed.com.cn;

xuajing@xinhuamed.com.cn

${ }^{\dagger}$ Jing Ma and Xiaoyuan Xu contributed equally to this work.

${ }^{4}$ Emergency Department, Xinhua Hospital, Shanghai Jiaotong University

School of Medicine, Shanghai 200092, P. R. China

'Department of Pharmacy, Xinhua Hospital, Shanghai Jiaotong University

School of Medicine, Shanghai 200092, P. R. China

Full list of author information is available at the end of the article
}

(C) The Author(s). 2020 Open Access This article is licensed under a Creative Commons Attribution 4.0 International License, which permits use, sharing, adaptation, distribution and reproduction in any medium or format, as long as you give appropriate credit to the original author(s) and the source, provide a link to the Creative Commons licence, and indicate if changes were made. The images or other third party material in this article are included in the article's Creative Commons licence, unless indicated otherwise in a credit line to the material. If material is not included in the article's Creative Commons licence and your intended use is not permitted by statutory regulation or exceeds the permitted use, you will need to obtain permission directly from the copyright holder. To view a copy of this licence, visit http://creativecommons.org/licenses/by/4.0/ The Creative Commons Public Domain Dedication waiver (http://creativecommons.org/publicdomain/zero/1.0/) applies to the data made available in this article, unless otherwise stated in a credit line to the data. 
(Continued from previous page)

Conclusion: Carnosine treatment protected against LPS injury by decreasing oxidative stress and inhibiting apoptosis through the p38 MAPK pathway.

Keywords: Carnosine, Caenorhabditis elegans, Lipopolysaccharide, Oxidative stress, p38 MAPK

\section{Background}

Lipopolysaccharide (LPS) is a pathogen-associated molecular pattern of gram-negative bacteria that is essential for its pathogenicity [1]. LPS plays an important role in the pathogenesis of sepsis, which is a systemic inflammatory response syndrome triggered by infections of bacteria, viruses, or parasites or by toxic products and has become a major cause of mortality in intensive care units. The characteristic features of sepsis include amplification of the initial inflammatory response followed by immunosuppression and multiple organ dysfunction or failure leading to death $[2,3]$. Studies have demonstrated that LPS is a main mediator of sepsis. LPS exposure may lead to an imbalanced immune response by eliciting the release of inflammatory mediators. In addition, LPS may lead to deregulated immune responses, triggering sepsis and consequently resulting in multiple organ failure [4-6].

Invertebrate models developed over the past decade have reduced the cost and complexity of mammalian assays and avoided the need for study review by institutional animal care and use committees [7]. Caenorhabditis elegans is a nematode used as a successful invertebrate model of pathogenesis and one of the most commonly used standard laboratory models for bacterial pathogenesis [8]. C. elegans has a simple lifecycle and short generation time and requires inexpensive and simple growth conditions [9]. Moreover, as nematodes have a complex innate immune system involving various signaling pathways and antimicrobial proteins and peptides, a wide range of mutant strains of C. elegans provides the opportunity to explore the molecular genetic determinants of pathogen toxicity [10]. C. elegans do not have an obvious acquired immune response, homologs of vertebrate cytokines and circulating immune cells. However, C. elegans possess many innate immune components that are evolutionarily conserved with vertebrates, including a single toll-like receptor and p38 mitogenactivated protein kinase (MAPK), mediating responses to infection [11]. LPS modulated the expression of selected host immune and aging-related genes in C. elegans $[1,12]$. In the present study, C. elegans with LPS-induced injury were used to conserve the mechanisms of innate immunity and stress response.

Carnosine ( $\beta$-alanyl-L-histidine; Fig. 1$)$ is a natural dipeptide that is usually abundant in excitable tissues such as nerves and muscles. Beyond its association with specific diseases, carnosine has been assigned many putative actions, such as free radical scavenging, anti-inflammation, and mobile organic $\mathrm{pH}$ buffering. Carnosine has in part been studied to determine its effect on oxidative stress conditions in vivo and in vitro [13-16]. Furthermore, some studies suggest that carnosine has a wholesome effect on the reduction of apoptosis by inhibiting caspase 3 increase and recovering $\mathrm{Bcl}-2$ level [17]. Carnosine showed protective effects on acute lung injury in sepsis rats by enhancing the antioxidant status along with a decrease in pro-inflammatory cytokines [18]. It has been shown to have beneficial effects in reducing acute kidney injury due to septic shock in a rat model of septicemia [19]. Carnosine may be an effective treatment for oxidative damage due to liver tissue perfusion defects in cases of septic shock [20]. However, the antioxidative and antiinflammatory effects of carnosine on C. elegans have not yet been determined.

The aim of the current study was to investigate the effect of carnosine on LPS-induced injury in C. elegans. We determined whether carnosine could effectively prevent LPS-induced injury in C. elegans. In addition, we elucidated the mechanism of carnosine suppression via the p38 MAPK signaling pathway and evaluated the therapeutic effect of carnosine.

\section{Methods}

\section{C. elegans culture}

C. elegans $\mathrm{N} 2$ (wild-type), sek-1 (km2) mutants, and pmk1 (km25) mutants were obtained from the Caenorhabditis

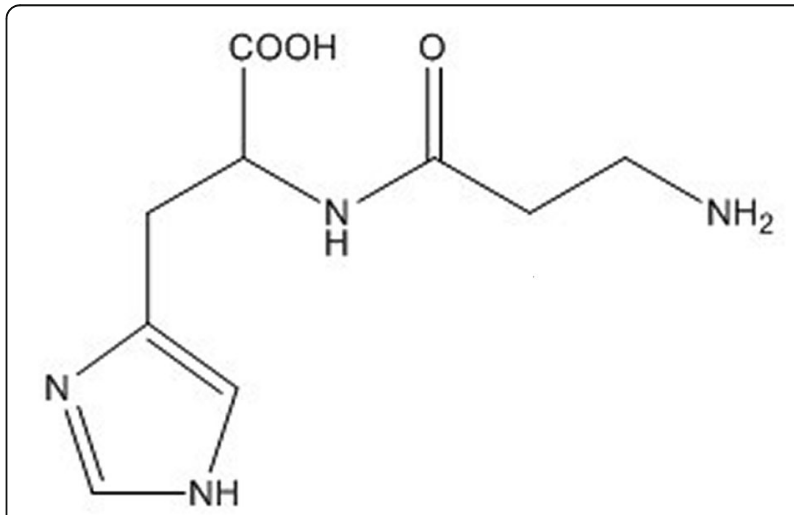

Fig. 1 The chemical structure of carnosine 
Genetics Center (CGC; University of Minnesota). The worms were cultured as follows: streptomycin-resistant variant strain Escherichia coli OP50 (OP50-1, obtained from CGC) was used as the food source for C. elegans. All worms were grown at $20^{\circ} \mathrm{C}$ on NGM plates $\left(1 \mathrm{mM} \mathrm{CaCl}_{2}\right.$, $1 \mathrm{mM} \mathrm{MgSO}_{4}, 5 \mathrm{~g} / \mathrm{mL}$ cholesterol, $50 \mathrm{mM} \mathrm{KH}_{2} \mathrm{PO}_{4} \mathrm{pH}$ 6.0, $25 \mathrm{mM} \mathrm{NaCl}, 1.7 \%$ agar, and $2.5 \mathrm{mg} / \mathrm{mL}$ peptone) with fresh OP50-1 as a food source.

\section{C. elegans experimental design}

L4 C. elegans larvae were maintained on NGM agar plates containing OP50-1 at $20^{\circ} \mathrm{C}$. Treatment of C. elegans with carnosine or M9 buffer $(5 \mathrm{~g} / \mathrm{L} \mathrm{NaCl}, 3 \mathrm{~g} / \mathrm{L}$ $\mathrm{KH}_{2} \mathrm{PO}_{4}, 6 \mathrm{~g} / \mathrm{L} \quad \mathrm{Na}_{2} \mathrm{HPO}_{4}, 0.25 \mathrm{~g} / \mathrm{L} \quad \mathrm{MgSO}_{4} 7 \mathrm{H}_{2} \mathrm{O}$, vehicle) was performed in liquid NGM without OP50. $C$. elegans individuals $(\mathrm{N} 2, \mathrm{~km} 25$, and $\mathrm{km} 2)$ were exposed to M9 buffer or carnosine $(0.1,1$, or $10 \mathrm{mM}) 30 \mathrm{~min}$ before LPS (serotype 055:B5; Sigma, Deisenhofen, Germany) treatment. The worms were then exposed to M9 buffer or LPS $(100 \mu \mathrm{g} / \mathrm{mL})$ for $24 \mathrm{~h}$, while carnosine was left in the buffer as a co-treatment with LPS. After $24 \mathrm{~h}$, C. elegans individuals were washed with M9 buffer and transferred to NGM agar plates, and this step was repeated twice. They were then allowed to acclimate for $30 \mathrm{~min}$ before performing survival assays. Data were obtained from five independent experiments.

\section{Behavioral assays}

Omega turn (a maneuver during which the body of the worm briefly resembles the Greek letter) and reversal tests were used to evaluate the behavior of $C$. elegans. Behavioral assays were performed on foodless NGM assay plates $(60-\mathrm{mm}$ diameter) [21]. Observation began 1 min after transfer to a new plate. The $C$. elegans individuals could be observed as they moved across the plate. The omega turn was defined as a sharp with a turning angle was greater than $135^{\circ}$ with three or more body bends. Reversals are a general and important feature of the worm's behavioral repertoire, ranging from aging and chemotaxis to escape from noxious stimuli, defined as a turn in which the head is in contact with the tail. Each animal was subjected to a maximum of five touches, and the first omega turn was scored under the observation of a stereomicroscope. A reversal was scored as any backward movement of the entire body was scored as a reversal. A SZX16 stereomicroscope (Olympus, Tokyo) was used for direct observation of behavior.

\section{RNA extraction and reverse transcription polymerase chain reaction}

The worms were collected after exposure to M9 buffer or LPS for $24 \mathrm{~h}$. Reverse transcription polymerase chain reaction (RT-PCR) was performed to verify the differential expression of sod-1, sod-2, sod-3, daf-16, ced-3, ced-
9, sek-1, and pmk-1. According to the manufacturer's instructions, total RNA was extracted using Trizol. In addition, according to the manufacturer's protocol, $5 \mathrm{mg}$ RNA was used to synthesize the first strand of complementary DNA (cDNA) using SuperScript II RNase HReverse Transcriptase (Invitrogen, Carlsbad, CA, USA) and used as template in subsequent PCR reactions with thermal cycling performed using an Eppendorf Mastercycler (Eppendorf, Germany). A Nano Drop 2000 spectrophotometer (Thermo, USA) was used to determine the concentration of total RNA, and RNA purity was assessed by OD260/OD280 ratios. The complementary DNA (cDNA) was amplified by using RT-PCR. Primer design is a common operation and primer amplification template is known, as following primer pairs: sod-1: (F) 5' -TGTCGAACCGTGCTGTCGCT-3' (R) 5' -TGGA CCGGCAGAAATGCATCCG-3'; sod-2: (F) 5'-ACCA TCGGCGGAGTTGCTCA-3', (R) 5' -AGCGTGCTCC CAGACGTCAA-3'; sod-3: (F) 5' -GTGGTGGACACATCAATC-3', (R) 5'-AAGTGGGACCATTCCTTC-3'; daf-16: (F) 5'-GGAAGAACTCGATCCGTCACA-3', (R) 5'-GATTCCTTCCTGGCTTTGCA-3'; sek-1: (F) 5' TGCTCAACGAGCTAGACG-3', (R) 5'-ATGTTCGA CGGTTTCACG-3'; pmk-1: (F) 5'-CGACTCCACGAG AAGGAT-3', (R) 5'-ATATGTACGACGGGCATG-3'; ced-3: (F) 5'-ACGGGAGATCGTGAAAGC-3', (R) 5'AGAGTTGGCGGATGAAGG-3'; ced-9: (F) 5'-AAAG GCACAGAGCCCACC-3', (R) 5'-CGTTCCCATAACT CGCATC-3'; and $\beta$-actin: (F) 5'-CCAGGAATTGCTGA TCGTATGCAGAA-3', (R) 5' -TGGAGAGGGAAGCGAGGATAGA-3'. The cycle number was determined from a linear amplification curve to ensure that amplification was within the linear amplification range. The level of $\beta$-actin expression was used to normalize the gene expression. By analyzing dissociation curves, each reaction was verified to contain a single amplification product. All sample reactions were performed three times. The 2$\Delta \Delta \mathrm{Ct}$ method, where $\Delta \Delta \mathrm{Ct}=\left[\left(\mathrm{Ct}_{\text {target }}-\mathrm{Ct}_{\text {actin }}\right)\right.$ Sample $]-$ $\left[\left(\mathrm{Ct}_{\text {target }}-\mathrm{Ct}_{\text {actin }}\right)\right.$ Control] with $\beta$-actin used as a reference gene were used to determine the relative changes in gene expression were determined using.

\section{Western blotting analysis}

C. elegans worms were collected and homogenized in icecold lysis buffer containing $50 \mathrm{mM}$ Tris- $\mathrm{HCl}, 1 \% \mathrm{NP}-40$, $150 \mathrm{mM} \mathrm{NaCl}, 2 \mathrm{mM}$ EDTA, and $1 \mathrm{mM} \mathrm{Na}_{3} \mathrm{VO}_{4}$ (pH 7.4) using a homogenizer (Bertin Precellys 24 Technologies) after exposure to M9 buffer or LPS for $24 \mathrm{~h}$. Protein samples were separated on $12 \%$ sodium dodecyl sulfate (SDS)-polyacrylamide gels and electro-transferred onto nitrocellulose membranes. The membranes were blocked by $5 \%$ fat-free milk, then incubated with primary antibodies against SEK1 (1:1000; Cell Signaling Technology, USA), p-p38 (1:1000; Abcam, UK), Bcl-2 (1:1000; Cell 
Signaling Technology), c-caspase3 (1:750; Cell Signaling Technology), and GAPDH (1:5000; Kangchen Bio-tech, Shanghai) for $2 \mathrm{~h}$ at room temperature. The membranes were washed three times with tris-buffered saline and Tween 20 buffer and incubated with IRDye 800 antirabbit Molecular Probe (1:8000; LI-COR Biosciences) or IRDye700 anti-mouse Molecular Probe (1:3000; LI-COR Biosciences, USA) for $2 \mathrm{~h}$. Images were acquired using an Odyssey Infrared Imaging System and analyzed using Odyssey software [22].

\section{Evaluation of antioxidant indices}

C. elegans worms were collected and homogenized in icecold lysis buffer after exposure to M9 buffer or LPS for 24 $\mathrm{h}$ and washed three times with M9 buffer as described above. The supernatant was centrifuged by ultrasonication. The activity of superoxide dismutase (SOD), glutathione reductase (GR), catalase (CAT) and the level of glutathione (GSH) or malondialdehyde (MDA) were detected following the instructions of T-SOD, GR, CAT, GSH or MDA assay kit (Nanjing Jiancheng Bioengineering Institute, Nanjing, China). The manipulation process was performed according to the manufacturer's instructions.

\section{Statistical analysis}

Statistical analyses were performed using SPSS 19.0 for Windows software. Data are presented as the mean \pm standard error of the mean (SEM) and included one-way analysis of variance followed by the least significant difference or Dunnett's T3 post-hoc test for multiple comparisons where equal variances were not assumed.

\section{Results}

Effect of carnosine on C. elegans in the presence of LPS Treatment with LPS $(100 \mu \mathrm{g} / \mathrm{mL})$ significantly decreased survival $(61.16 \%)$ compared with that of non-treated worms (vehicle group). Carnosine significantly increased survival when treated at concentrations of $1 \mathrm{mM}(P<$ $0.01)$ and $10 \mathrm{mM}(P<0.001)$ compared with the vehicle group. Survival was also increased in the $0.1 \mathrm{mM}$ carnosine group but failed to reach a significant difference compared with that in the vehicle group (Fig. 2a). LPS exposure caused a significant reduction in the frequency of reversals (Fig. 2b) and omega turns (Fig. 2c). However, 1 and $10 \mathrm{mM}$ carnosine pre-treatments significantly elevated the frequency of the behavior of C. elegans compared with LPS treatment. These changes were not obvious with pre-treatment with low concentrations of carnosine.

Carnosine inhibited apoptosis induced by LPS in C. elegans Western blotting results showed that the level of cleaved caspase 3 protein was significantly elevated in the LPStreated group $(P<0.05)$, and this increased expression was down-regulated by treatment with 1 and $10 \mathrm{mM}$ carnosine (Fig. 3a). In contrast, the levels of Bcl-2 expression were significantly decreased by exposure to LPS $(P<0.01)$, but treatment with carnosine effectively elevated the levels of Bcl-2 expression in LPS-treated worms (Fig. 3b). The effect of carnosine on gene expression of the apoptosis inhibitor ced-9 (the Bcl-2 homolog) and ced-3 (the initiator procaspase) was evaluated by using RTPCR. As shown in Fig. 3c and d, compared with the control group, ced-9 levels were significantly decreased and ced-3 levels were significantly increased in the LPS group. Carnosine effectively reversed LPS-induced changes in gene expression of ced-9 and ced-3 $(P<0.001)$.

\section{Carnosine ameliorated oxidative stress induced by LPS in C. elegans}

The levels of SOD, CAT, GSH, and GR were significantly decreased in LPS group compared with those in the vehicle group $(P<0.001)$. However, treatment with carnosine effectively elevated these levels in C. elegans treated with LPS (Fig. 4a-d). The levels of MDA in $C$. elegans were increased by LPS treatment $(P<0.001)$, but this was significantly reversed by pre-treatment with carnosine (Fig. 4e). The levels of $\operatorname{sod}-1$, sod-2, sod-3, and daf-16 mRNA in C. elegans decreased in response to LPS treatment (Fig. 4f-i), but these were significantly reversed by carnosine treatment.

\section{Effect of carnosine on p38 MAPK signaling pathway of $C$. elegans treated with LPS}

After exposure to LPS for $24 \mathrm{~h}$, the transcriptional expression of genes pmk-1 and sek-1 significantly increased compared with that in the vehicle group. However, treatment with carnosine effectively reduced $p m k-1$ and $s e k-1$ gene expression in worms treated with LPS (Fig. 5a and b). As shown in Fig. $5 \mathrm{c}$, the survival rate of worms was not altered by LPS treatment in pmk-1 (km25) mutants or sek-1 $(\mathrm{km} 4)$ mutants, suggesting that pmk-1 and sek-1 may play pivotal roles in mediating LPS-induced death in C. elegans. Compared with that in the vehicle group, both phosphorylated p38 (p-p38) and SEK1 expression was greatly increased in the LPS group of $C$. elegans worms. Nonetheless, the expression of p-p38 and SEK1 was reduced following pretreatment with carnosine (Fig. $5 \mathrm{~d}$ and e).

\section{Discussion}

The present study evaluated the potential of carnosine as an antioxidant agent using a C. elegans model. Carnosine increased the survival rates and frequency of normal behavior of worms treated with LPS group significantly. We found that carnosine significantly attenuated oxidative injury and prevented apoptosis. The protective effect of carnosine on C. elegans may be related to the p38 MAPK signaling pathway. 


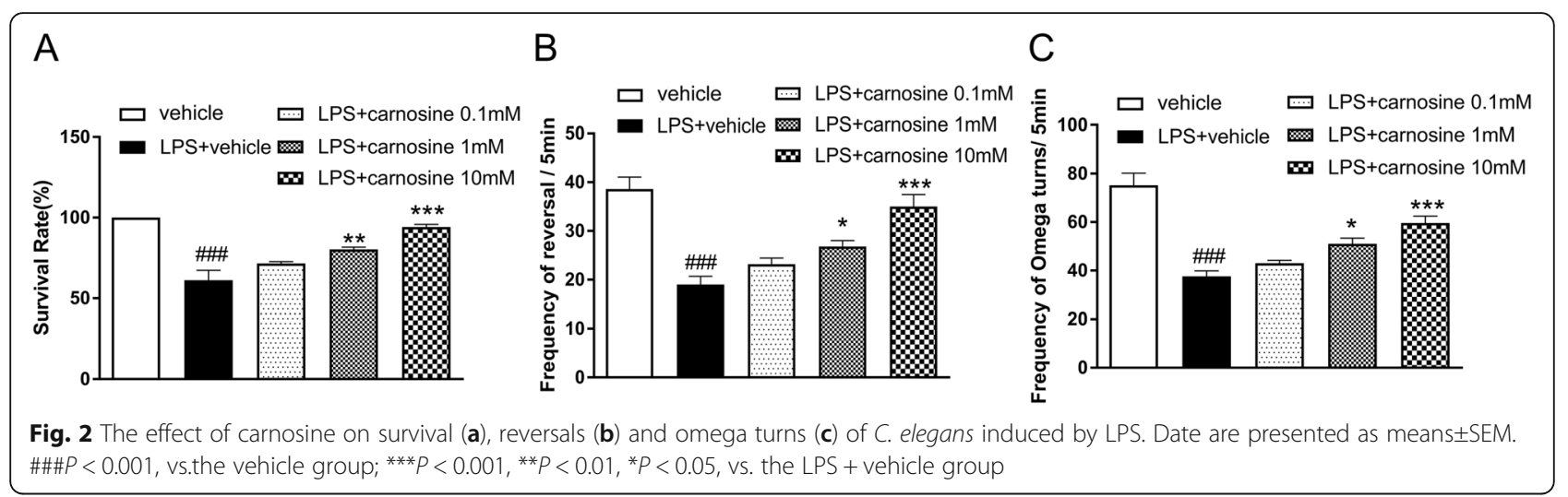

Carnosine has been reported to possess antiinflammatory and antioxidative properties [13]. However, the effect of carnosine on LPS-induced injury in $C$. elegans has not been reported. In the current study, LPS exposure resulted in significant nematode mortality and reduced frequencies of reversals and omega turns, which were effects rescued by carnosine. As an important enzyme that catalyzes the dismutation of superoxide, SOD plays an important role in the first defense line of cell oxidative damage [23]. Using CAT, SOD can convert ROS into hydrogen peroxide, resulting in an oxidant effect [24]. GSH is an important biological free radical scavenger and antioxidant and maintained endogenous redox homeostasis [25]. GR is also an important enzyme that maintains the levels of reduced GSH in cells [26], while MDA is an end product of polyunsaturated fatty
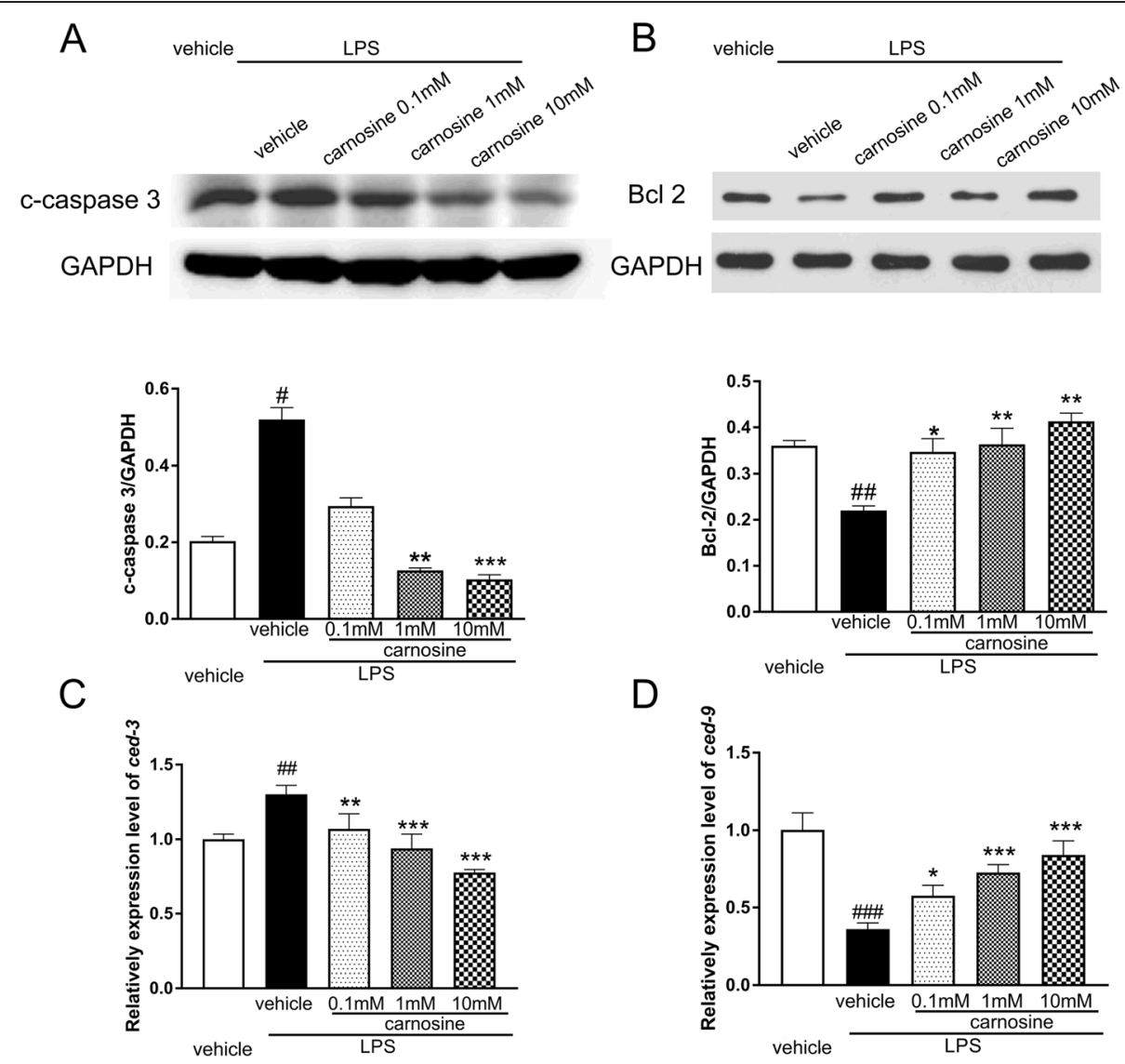

Fig. 3 Effects of carnosine on the apoptosis. Western blot analysis of c-caspase 3 (a) and Bcl-2 (b) protein levels in C. elegans after LPS exposure. PCR annlysis of ced-3 (c) and ced-9 (d) gene expression in C. elegans after LPS exposure. Date are presented as means \pm SEM. \#\#\#P<0.001, \#\#P< 0.01 , $\# P<0.05$, vs. the vehicle group; ${ }^{* *} P<0.001,{ }^{* *} P<0.01,{ }^{*} P<0.05$, vs. the LPS + vehicle group 

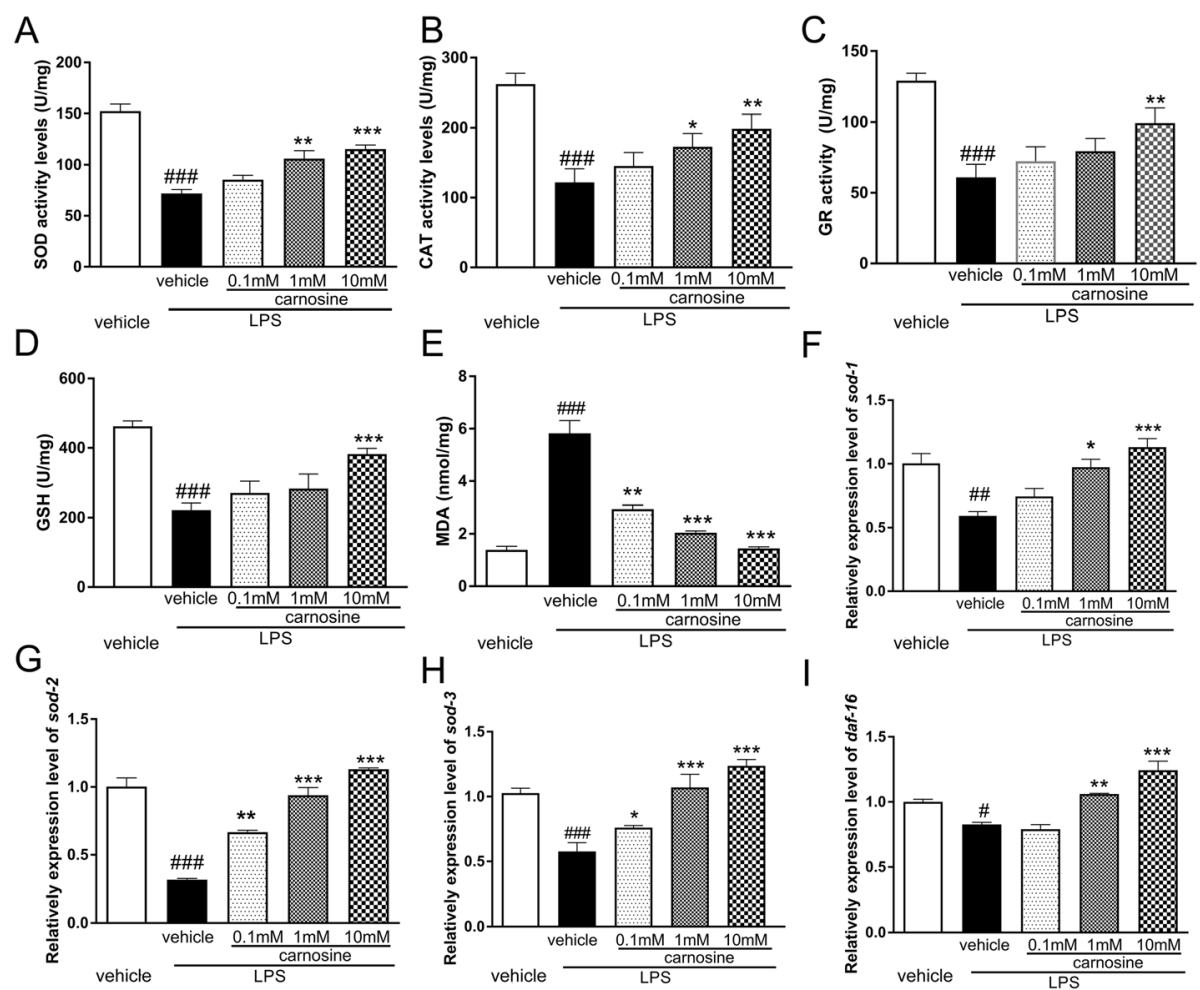

Fig. 4 Carnosine ameliorates oxidative stress in C. elegans induced by LPS. a The levels of SOD (a), CAT (b), GSH (c), GR (d) and MDA (e) were detected in C. elegans after LPS exposure. The levels of sod-1 (f), sod-2 (g), sod-3 (h) and daf-16 (i) mRNA in C. elegans were decreased by LPS treatment, and were significantly reversed by carnosine. Date are presented as means \pm SEM. \#\#\#P<0.001, vs. the vehicle group; ${ }^{* * *} P<0.001$, ${ }^{* *} P<0.01$, ${ }^{*} P<0.05$, vs. the $L P S+$ vehicle group

acid peroxidation, which is promoted by oxidative stress [27]. We compared the levels of important enzymes in C. elegans worms following treatment with LPS and carnosine. MDA levels were markedly increased and GSH levels were decreased in C. elegans treated with LPS, demonstrating the occurrence of oxidative stress. The activities of various enzymes regulating oxidative stress, including SOD, GR, and CAT, were significantly decreased following exposure to LPS. The administration of carnosine ameliorated LPS-induced oxidative stress, as evidenced by the significant inhibition on LPSinduced increase of MDA and decrease of SOD, GR, CAT, and GSH. The transcription factor daf-16 is a homologous gene of mammal FOXO proteins in C. elegans that play critical roles in stress response [28]. Daf16-knockout worms are highly sensitive to oxidative stress, and they exhibit decreased lifespan $[29,30]$. The mammalian ortholog of SIRT1, the sir-2.1 gene, is known to modulate oxidative stress responses and longevity through daf-16 [31, 32]. The antioxidative effect of carnosine was confirmed by the reversal of downregulation of three sod genes and daf-16. The dose of carnosine (0.1-10 $\mathrm{mM})$ in our research is similar to the dose in humans (0.5$2 \mathrm{~g} /$ day) at which carnosine exhibited protective effects on LPS-induced oxidative damage [33-35]. Caspase 3, a frequently activated death protease, catalyzes the specific cleavage of many key cellular proteins. For instance, caspase 3 is required for apoptotic scenarios in tissues and cells and is indispensable for DNA fragmentation and apoptotic chromatin condensation [36]. Meanwhile, Bcl-2, an antiapoptotic protein, sequesters and inhibits pro-apoptotic proteins and prevents apoptosis by blocking the key steps of caspases activation [37]. Carnosine effectively elevated the expression levels of Bcl-2 and reduced the levels of caspase 3 in C. elegans worms treated with LPS. The C. elegans ced-3 gene is essential for apoptosis and encodes apoptotic caspases [38]. ced-3 is a homolog of mammalian caspase 3, which is a protease that eventually kills cells. The activation and regulation of ced-3 during apoptosis plays important roles in the activation and function of caspases and apoptosis. In contrast to ced-3, C. elegans Bcl-2 homolog ced-9 plays a central role in preventing cell death in worms [39]. Carnosine effectively reversed the decrease of ced-9 and increase of ced-3 induced by LPS. Our results from both behavioral and western blotting analyses show that LPS-induced apoptosis in C. elegans could be rescued by carnosine.

It is also known that p38 MAPK plays a key role in the process of sepsis caused by LPS [40]. As a member of the MAPK superfamily, p38 is activated by various 


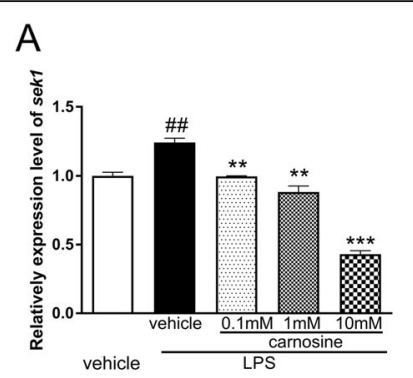

D
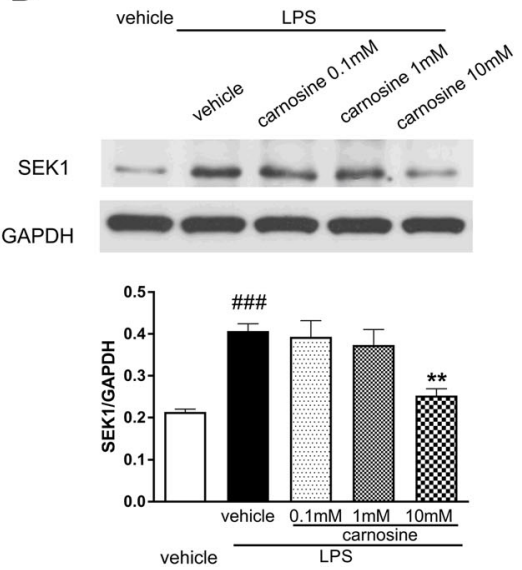

C
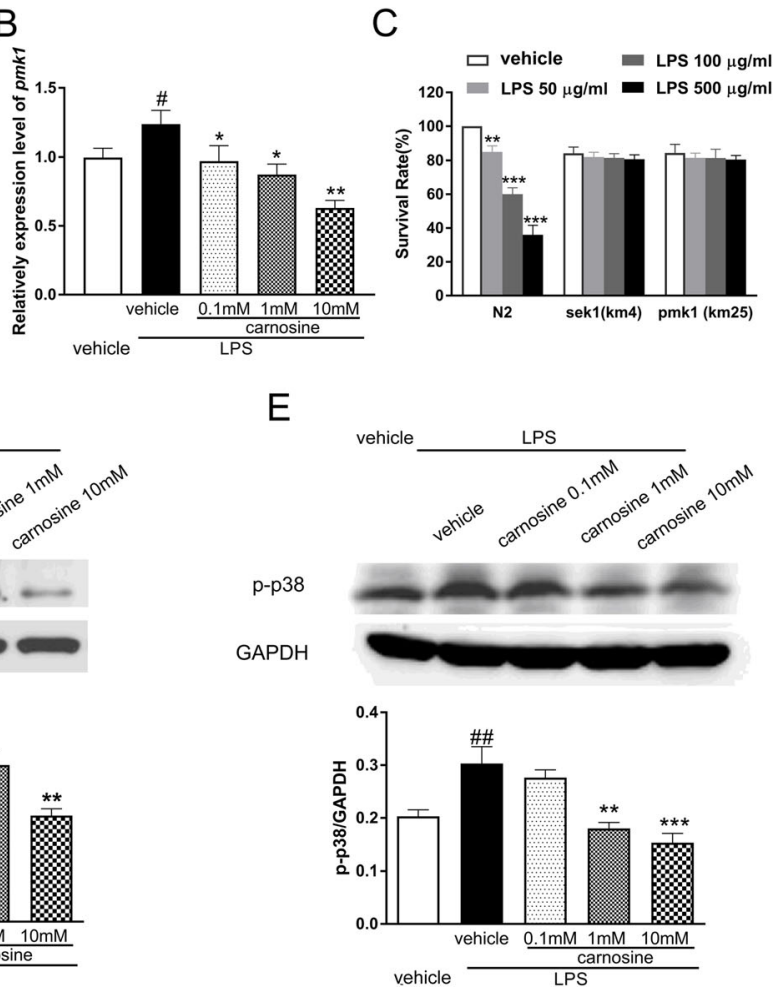

Fig. 5 Effects of carnosine on the expression of p38 MAPK signaling pathway. The levels of sek-1 (a) and pmk-1 (b) mRNA in C. elegans were detected by PCR. c The survival rate of worms were not altered by LPS treatment in pmk-1(km25), sek-1(km4) mutants. Western blot analysis of SEK1 (d) and p-p38 (e) protein levels in C. elegans after LPS exposure. Date are presented as means \pm SEM. \#\#\#P<0.001, \#\#P<0.01, \#P<0.05, vs.the vehicle group; ${ }^{* *} P<0.001,{ }^{*} P<0.01,{ }^{*} P<0.05$, vs. the LPS + vehicle group

cellular stresses and ligands. In C. elegans, pmk-1 is a p38 MAPK homolog, which is associated with the apoptotic regulation of germ cells, innate immune response, and oxidative reactions [41, 42]. SEK-1 is also an important component of the p38 pathway, which is required for innate immunity in C. elegans [43]. SEK-1 is a MAPK kinase and the $C$. elegans ortholog of mammalian MKK3/MKK6 in the p38 MAPK pathway [44]. Our results from both PCR and western blotting analyses demonstrated that LPS significantly increased the transcriptional expression of $p m k-1$ and sek-1 genes and the expression level of p-p38 and SEK1. We therefore investigated the effects of carnosine on the local activities of the p38 MAPK signaling pathway in LPS-induced C. elegans. The results showed that carnosine inhibited the expression of the p38 MAPK signaling pathway. Therefore, carnosine might act as a suppressor of the p38 MAPK signaling pathway. To confirm the function of the p38 MAPK signaling pathway in regulating LPS toxicity, we investigated LPS toxicity in worms with mutations in pmk-1 $(\mathrm{km} 25)$ and sek-1 $(\mathrm{km} 4)$, which are genes associated with the p38 MAPK signaling pathway in nematodes. Our results revealed that $p m k-1$ and sek-1 play pivotal roles in mediating LPS-induced death in $C$. elegans, which further confirmed that carnosine protected the nematodes from LPS-induced damage through the p38 MAPK signaling pathway.

\section{Conclusions}

In conclusion, our study reveals a potential protective role of carnosine in LPS-induced C. elegans model. Carnosine treatment protected against LPS-induced injury by decreasing oxidative stress and inhibiting apoptosis through the p38 MAPK pathway. Our findings provide insights into the impact of carnosine on conserved mechanisms of innate immunity and stress response.

\section{Supplementary Information}

The online version contains supplementary material available at https://doi. org/10.1186/s40360-020-00455-w.

\section{Additional file 1.}

\section{Abbreviations}

CAT: Catalase; cDNA: complementary DNA; C. elegans: Caenorhabditis elegans; ELISA: Enzyme-linked Immunosorbent assay; GR: Glutathione reductase; GSH: Glutathione; LPS: Lipopolysaccharide; MAPK: Mitogen-activated protein kinase; MDA: Malondialdehyde; ROS: Reactive oxygen species; RT-

PCR: Reverse transcription polymerase chain reaction; SDS: Sodium dodecyl sulfate; SOD: Superoxide dismutase; TNF-a: Tumor necrosis factor alpha 


\section{Acknowledgements}

Not applicable.

\section{Authors' contributions}

SWJ and AJX contributed equally to this work. All experiments were performed by JM, XYX, and RRW. JM, HJY1, HJY2, and HMZ wrote the manuscript. JM and AJX were in charge of data analysis and technical graphics and helped revise the manuscript. HMZ and SWJ helped revise the manuscript. The authors read and approved the final manuscript.

\section{Funding}

This work was funded by the Natural Science Foundation of Shanghai (No.c18ZR1424900), the National Natural Science Foundation of China (81402904, 81500930), the Science and Technology Commission of Shanghai Municipality (No. 19401900500), the clinical research project of Shanghai Health and Family Planning Commission (No. 201840121), the Innovation program of Shanghai Health Commission [ZY (2018-2020)-ZWB-1001-CPJS1], and the Clinical Pharmacy Innovation Research Institute of Shanghai Jiao Tong University School of Medicine (CXYJY2019QN001).

\section{Availability of data and materials}

All data generated and analyzed during this study are included in this article. The datasets used and/or analyzed during the current study are available from the corresponding author on reasonable request.

\section{Ethics approval and consent to participate}

All animal experimental procedures in the present study were performed under protocols approved by the Institutional Animal Care and Use Committee of Shanghai Jiaotong University School of Medicine. No permissions were necessary to collect the specimens in our study.

\section{Consent for publication}

Not applicable.

\section{Competing interests}

The authors declare that they have no conflict of interest.

\section{Author details}

'Department of Pharmacy, Xinhua Hospital, Shanghai Jiaotong University School of Medicine, Shanghai 200092, P. R. China. ${ }^{2}$ Department of Endocrinology, Xinhua Hospital, Shanghai Jiaotong University School of Medicine, Shanghai 200092, P. R. China. ${ }^{3}$ Institute for metabolic and neuropsychiatric Disorders, Binzhou Medical University Hospital, Binzhou, China. ${ }^{4}$ Emergency Department, Xinhua Hospital, Shanghai Jiaotong University School of Medicine, Shanghai 200092, P. R. China.

Received: 29 April 2020 Accepted: 27 October 2020

Published online: 03 December 2020

\section{References}

1. Sivamaruthi BS, Prasanth MI, Balamurugan K. Alterations in Caenorhabditis elegans and Cronobacter sakazakii lipopolysaccharide during interaction. Arch Microbiol. 2015;197(2):327-37.

2. Rello J, Valenzuela-Sanchez F, Ruiz-Rodriquez M, Moyano S. Sepsis: a review of advances in management. Adv Ther. 2017;34(11):2393-411

3. Fernando SM, Rochwerg B, Seely AJE. Clinical implications of the third international consensus definitions for Sepsis and septic shock (Sepsis-3). CMAJ. 2018:190(36):E1058-9.

4. Seeley JJ, Ghosh S. Molecular mechanisms of innate memory and tolerance to LPS. J Leukoc Biol. 2017:101(1):107-19.

5. Dickson K, Lehmann C. Inflammatory Response to Different Toxins in Experimental Sepsis Models. Int J Mol Sci. 2019;20(18).

6. Cavaillon JM. Exotoxins and endotoxins: inducers of inflammatory cytokines. Toxicon. 2018;149:45-53

7. Pir GJ, Choudhary B, Mandelkow E. Caenorhabditis elegans models of tauopathy. FASEB J. 2017;31(12):5137-48

8. Maynard C, Weinkove D. Bacteria increase host micronutrient availability: mechanisms revealed by studies in C. elegans. Genes Nutr. 2020;15(1):4.

9. Khan F, Jain S, Oloketuyi SF. Bacteria and bacterial products: foe and friends to Caenorhabditis elegans. Microbiol Res. 2018;215:102-13.
10. Ermolaeva MA, Schumacher B. Insights from the worm: the C. elegans model for innate immunity. Semin Immunol. 2014;26(4):303-9.

11. Battisti JM, Watson LA, Naung MT, Drobish AM, Voronina E, Minnick MF. Analysis of the Caenorhabditis elegans innate immune response to Coxiella burnetii. Innate Immun. 2017;23(2):111-27.

12. Chan JP, Wright JR, Wong HT, Ardasheva A, Brumbaugh J, McLimans C, Lamendella R. Using bacterial Transcriptomics to investigate targets of hostbacterial interactions in Caenorhabditis elegans. Sci Rep. 2019;9(1):5545.

13. Ma J, Chen J, Bo S, Lu X, Zhang J. Protective effect of carnosine after chronic cerebral hypoperfusion possibly through suppressing astrocyte activation. Am J Transl Res. 2015;7(12):2706-15.

14. Fresta CG, Fidilio A, Lazzarino G, Musso N, Grasso M, Merlo S, Amorini AM, Bucolo C, Tavazzi B, Lazzarino G, Lunte SM, Caraci F, Caruso G. Modulation of Pro-Oxidant and Pro-Inflammatory Activities of M1 Macrophages by the Natural Dipeptide Carnosine. Int J Mol Sci. 2020;21(3).

15. Caruso G, Fresta CG, Fidilio A, O'Donnell F, Musso N, Lazzarino G, Grasso M, Amorini AM, Tascedda F, Bucolo C, Drago F, Tavazzi B, Lazzarino G, Lunte SM, Caraci F. Carnosine Decreases PMA-Induced Oxidative Stress and Inflammation in Murine Macrophages. Antioxidants (Basel). 2019; 8(8).

16. Ma J, Yan H, Wang R, Bo S, Lu X, Zhang J, Xu A. Protective effect of carnosine on white matter damage in corpus striatum induced by chronic cerebral hypoperfusion. Neurosci Lett. 2018;683:54-60.

17. Attia H, Fadda L, Al-Rasheed N, Al-Rasheed N, Maysarah N. Carnosine and Larginine attenuate the downregulation of brain monoamines and gamma aminobutyric acid; reverse apoptosis and upregulate the expression of angiogenic factors in a model of hemic hypoxia in rats. Naunyn Schmiedeberg's Arch Pharmacol. 2019:393(3):381-94.

18. Sun C, Wu Q, Zhang X, He Q, Zhao H. Mechanistic evaluation of the protective effect of Carnosine on acute lung injury in Sepsis rats. Pharmacology. 2017;100(5-6):292-300.

19. Sahin S, Burukoglu DD. Effects of Carnosine (Beta-Alanyl-L-Histidine) in an experimental rat model of acute kidney injury due to septic shock. Med Sci Monit. 2018:24:305-16.

20. Sahin S, Oter S, Burukoglu D, Sutken E. The effects of carnosine in an experimental rat model of septic shock. Med Sci Monit Basic Res. 2013;19:54-61.

21. Ma J, Wang R, Yan H, Xu R, Xu A, Zhang J. Protective effects of Baicalin on lipopolysaccharide-induced injury in Caenorhabditis elegans. Pharmacology. 2020;105(1-2):109-17.

22. Ma J, Zhang J, Hou WW, Wu XH, Liao RJ, Chen Y, Wang Z, Zhang XN, Zhang LS, Zhou YD, Chen Z, Hu WW. Early treatment of minocycline alleviates white matter and cognitive impairments after chronic cerebral hypoperfusion. Sci Rep. 2015;5:12079.

23. Wang Y, Branicky R, Noe A, Hekimi S. Superoxide dismutases: dual roles in controlling ROS damage and regulating ROS signaling. J Cell Biol. 2018; 217(6):1915-28.

24. He L, He T, Farrar S, Ji L, Liu T, Ma X. Antioxidants maintain cellular redox homeostasis by elimination of reactive oxygen species. Cell Physiol Biochem. 2017:44(2):532-53.

25. Yahfoufi N, Alsadi N, Jambi M, Matar C. The Immunomodulatory and AntiInflammatory Role of Polyphenols. Nutrients. 2018:10(11).

26. Hoffmann C, Dietrich M, Herrmann AK, Schacht T, Albrecht P, Methner A. Dimethyl Fumarate induces glutathione recycling by Upregulation of glutathione Reductase. Oxidative Med Cell Longev. 2017;2017:6093903.

27. Islam MT. Oxidative stress and mitochondrial dysfunction-linked neurodegenerative disorders. Neurol Res. 2017;39(1):73-82.

28. Jia W, Peng Q, Su L, Yu X, Ma C W, Liang M, Yin X, Zou Y, Huang Z. Novel Bioactive Peptides from Meretrix meretrix Protect Caenorhabditis elegans against Free Radical-Induced Oxidative Stress through the Stress Response Factor DAF-16/FOXO. Mar Drugs. 2018;16(11).

29. Kaletsky R, Lakhina V, Arey R, Williams A, Landis J, Ashraf J, Murphy CT. The C. elegans adult neuronal IIS/FOXO transcriptome reveals adult phenotype regulators. Nature. 2016;529(7584):92-6.

30. Gusarov I, Pani B, Gautier L, Smolentseva O, Eremina S, Shamovsky I, Katkova-Zhukotskaya O, Mironov A, Nudler E. Glycogen controls Caenorhabditis elegans lifespan and resistance to oxidative stress. Nat Commun. 2017:8:15868.

31. Tepper RG, Ashraf J, Kaletsky R, Kleemann G, Murphy CT, Bussemaker HJ. PQM-1 complements DAF-16 as a key transcriptional regulator of DAF-2 mediated development and longevity. Cell. 2013;154(3):676-90.

32. Kenyon CJ. The genetics of ageing. Nature. 2010;464(7288):504-12. 
33. Regazzoni L, de Courten B, Garzon D, Altomare A, Marinello C, Jakubova M, Vallova S, Krumpolec P, Carini M, Ukropec J, Ukropcova B, Aldini G. A carnosine intervention study in overweight human volunteers: bioavailability and reactive carbonyl species sequestering effect. Sci Rep. 2016;6:27224.

34. Houjeghani S, Kheirouri S, Faraji E, Jafarabadi MA. I-Carnosine supplementation attenuated fasting glucose, triglycerides, advanced glycation end products, and tumor necrosis factor-alpha levels in patients with type 2 diabetes: a double-blind placebo-controlled randomized clinical trial. Nutr Res. 2018:49:96-106.

35. Mehrazad-Saber Z, Kheirouri S, Noorazar SG. Effects of I-Carnosine supplementation on sleep disorders and disease severity in autistic children: a randomized, controlled clinical trial. Basic Clin Pharmacol Toxicol. 2018; 123(1):72-7.

36. Julien O, Wells JA. Caspases and their substrates. Cell Death Differ. 2017; 24(8):1380-9.

37. Siddiqui WA, Ahad $\mathrm{A}$, Ahsan $\mathrm{H}$. The mystery of $\mathrm{BCL} 2$ family: $\mathrm{BCL}-2$ proteins and apoptosis: an update. Arch Toxicol. 2015;89(3):289-317.

38. Brantley SJ, Cotten SW, Lamson DR, Smith GR, Liu R, Williams KP. Discovery of small molecule inhibitors for the C. elegans caspase CED-3 by highthroughput screening. Biochem Biophys Res Commun. 2017:491(3):773-9.

39. Modi V, Sankararamakrishnan R. Antiapoptotic BCl-2 homolog CED-9 in Caenorhabditis elegans: dynamics of BH3 and CED-4 binding regions and comparison with mammalian antiapoptotic BCl-2 proteins. Proteins. 2014; 82(6):1035-47.

40. Li L, Liu Y, Chen HZ, Li FW, Wu JF, Zhang HK, He JP, Xing YZ, Chen Y, Wang WJ, Tian XY, Li AZ, Zhang Q, Huang PQ, Han J, Lin T, Wu Q. Impeding the interaction between Nur77 and p38 reduces LPS-induced inflammation. Nat Chem Biol. 2015;11(5):339-46.

41. Zhao Y, Zhi L, Wu Q, Yu Y, Sun Q, Wang D. p38 MAPK-SKN-1/Nrf signaling cascade is required for intestinal barrier against graphene oxide toxicity in Caenorhabditis elegans. Nanotoxicology. 2016;10(10):1469-79.

42. Cheesman HK, Feinbaum RL, Thekkiniath J, Dowen RH, Conery AL, PukkilaWorley R. Aberrant Activation of p38 MAP Kinase-Dependent Innate Immune Responses Is Toxic to Caenorhabditis elegans. G3 (Bethesda). 2016; 6(3):541-9.

43. Andrusiak MG, Jin Y. Context specificity of stress-activated mitogenactivated protein (MAP) kinase signaling: the story as told by Caenorhabditis elegans. J Biol Chem. 2016;291(15):7796-804.

44. Tanaka-Hino M, Sagasti A, Hisamoto N, Kawasaki M, Nakano S, NinomiyaTsuji J, Bargmann Cl, Matsumoto K. SEK-1 MAPKK mediates Ca2+ signaling to determine neuronal asymmetric development in Caenorhabditis elegans. EMBO Rep. 2002;3(1):56-62.

\section{Publisher's Note}

Springer Nature remains neutral with regard to jurisdictional claims in published maps and institutional affiliations.

Ready to submit your research? Choose BMC and benefit from:

- fast, convenient online submission

- thorough peer review by experienced researchers in your field

- rapid publication on acceptance

- support for research data, including large and complex data types

- gold Open Access which fosters wider collaboration and increased citations

- maximum visibility for your research: over $100 \mathrm{M}$ website views per year

At $\mathrm{BMC}$, research is always in progress.

Learn more biomedcentral.com/submissions 